\title{
MicroscopyAwards
}

\section{Microscopy Society of America Awards: 2021 Award Winners}

\author{
Miaofang Chi \\ Oak Ridge National Laboratory \\ chim@ornl.gov
}

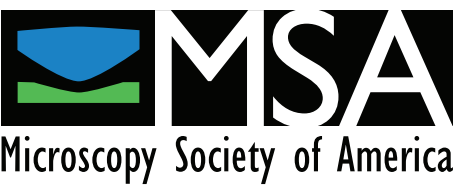

Each year, the Microscopy Society of America (MSA) provides several major awards for outstanding contributions to the fields of microscopy and microanalysis and service to the Society. While recipients of these awards are listed under the tab Awards \& Scholarships - Society Awards | Microscopy Society of America on the MSA homepage, little information as to why awards were bestowed is provided. This article highlights the contributions of the winners of the 2021 MSA major Society awards and the MSA Fellows. The information presented here represents a short summary of information provided in the awardees' nomination packages. Guidelines, including deadlines for nominating individuals for these and other MSA awards, can be found at https://www.microscopy.org/awards/society.cfm.

\section{Distinguished Scientist Awards}

Distinguished Scientist Awards annually recognize a preeminent senior scientist, from both the biological and physical sciences, who has a long-standing record of achievement during his or her career in the field of microscopy or microanalysis.

\section{Biological Sciences Distinguished Scientist: David Agard}

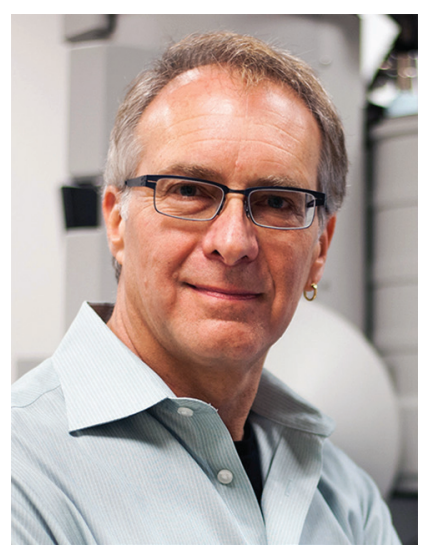

David Agard, Professor of Biochemistry \& Biophysics and Professor of Pharmaceutical Chemistry at the University of California, San Francisco.

David's group also pioneered software development for correcting beam-induced motion between frames of movies acquired using cryo-EM. These methods are used countless times each day in laboratories around the world.
David's major technical developments in microscopy have been critical to contributing to his fundamental interest in understanding the complex relationships between structure and function at both the molecular and cellular level. Having a solid background in structural biophysics, David's research focuses on elucidating the mechanisms of assisted folding by the Hsp90 molecular chaperone system, microtubule nucleation, and phage nucleus assembly. He has a very wide range of research interests including understanding the mechanisms of assisted protein folding and microtubule assembly, the structural basis for ligand specificity, and the architecture and function of cellular machines. He has been exceptionally productive throughout his career, as is evident from more than 260 publications. His work has been recognized by his election to the United States National Academy of Sciences in 2007 and the American Academy of Arts and Sciences in 2009.

\section{Physical Sciences Distinguished Scientists: Maximilian Haider and Knut W. Urban}
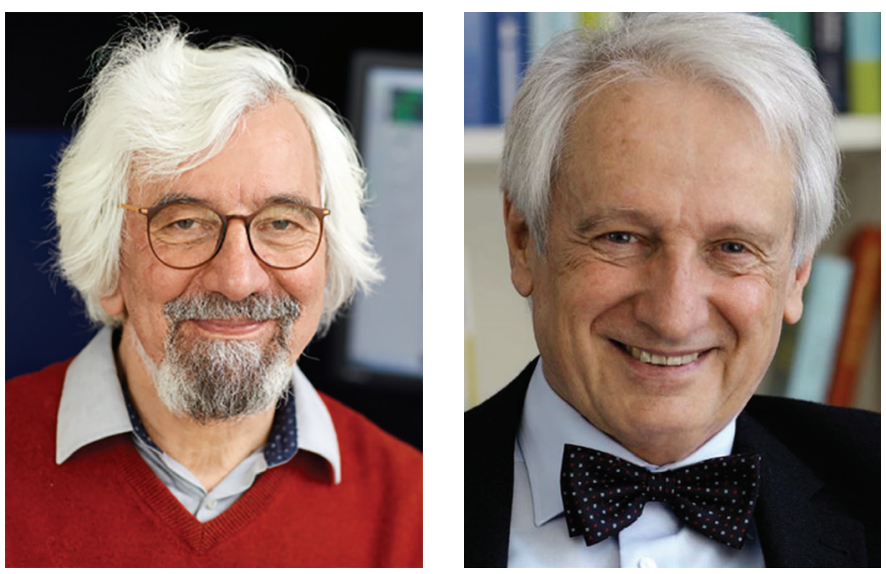

Left: Maximilian Haider, Group Leader, Physical Instrumentation Program, European Molecular Biology Laboratory. Right: Knut Urban, Forschungszentrum Jülich, Ernst Ruska-Center for Microscopy and Spectroscopy with Electrons.

Maximilian Haider is an Austrian physicist who was introduced to electron optics by Otto Scherzer and Harald Rose in Kiel and Darmstadt, Germany, where they developed a multipole-element for running the Cs-Cc aberration correction project. In 1987, as his $\mathrm{PhD}$ project on the "Design, construction, and testing of a corrected electron energy loss spectrometer with large dispersion and a large acceptance angle," he 
developed a new electron spectrometer. In 1989, he became Group Leader within the Physical Instrumentation Program at the European Molecular Biology Laboratory (EMBL) in Heidelberg. Although working in a biological environment, he convinced the laboratory to accept a project to develop a Cs-corrected TEM for high-resolution EM in cooperation with Rose, and for later applications with Knut Urban. Additional contributions include development of the first sextupole corrector, based on Rose's design, and implementation of the first aberration-corrected conventional transmission electron microscope.

In 1996 he founded, together with Joachim Zach, Corrected Electron Optical Systems GmbH in Heidelberg, for which he acted as managing director until 2015. In 2008, he was appointed as Honorary Professor at the Karlsruhe Institute of Technology, and together with Rose and Urban, he has received several honors and awards including the 2011 Israeli Wolf Research Prize for Physics and the 2013 Foundation Frontiers of Knowledge Award. In 2020, he received the Kavli Prize in Nanoscience with Ondrej Krivanek, Knut Urban, and Harald Rose.

Knut Urban studied at the University of Stuttgart, where he obtained his PhD in Physics in 1972. He then moved to the Max Planck Institute of Metals Research in Stuttgart, where he was appointed head of the high-voltage electron microscopy group. He spent extended research stays at CEA Saclay/Paris, BARC Mumbai, and Tohoku University, Sendai. In 1984, he was appointed Professor at the University of Erlangen and, in 1987, the Chair of Experimental Physics at RWTH Aachen University, and the Director of the Institute of Solid State Research, Forschungszentrum Jülich, Germany. In 2004, he became a co-founder of the Ernst Ruska-Center for Microscopy and Spectroscopy with Electrons. He has worked in many fields of experimental physics, including atomic defects in metals and alloys, the plasticity of quasicrystals, and the function of oxides. Urban has received numerous national and international awards, including the 2006 MRS Von Hippel Award, the 2008 Honda Prize for Ecotechnology, the 2011 Israeli Wolf Research Prize for Physics, and the 2020 Kavli Prize in Nanoscience. Urban was President of the German Physical Society from 2004 to 2006 and is highly recognized for his role in implementing the first aberration-corrected conventional transmission electron microscope.

\section{Burton Medal}

The Burton Medal honors annually the distinguished contributions to the field of microscopy and microanalysis by a scientist who is less than 40 years of age on January 1st of the award year (that is, the awardee to be honored at M\&M 2021 cannot have been born earlier than 1981).

\section{Burton Medal - Biological Sciences: Reto Fiolka}

Reto Fiolka obtained his $\mathrm{PhD}$ at the Institute of Nanotechnology at ETH in Zurich, Switzerland in 2009. He then conducted postdoctoral research at the Howard Hughes Medical Institute Janelia Research Campus where he worked on 3D-structured illumination microscopy and adaptive optics. In his current position at the University of Texas

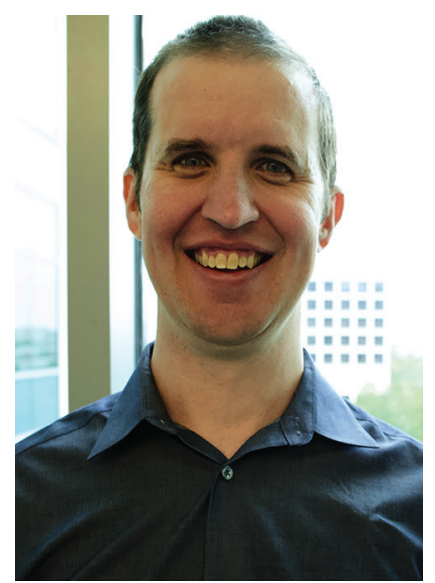

Southwestern, Reto has developed novel techniques in light-sheet microscopy and has provided several major breakthroughs in microscopy. With Field Synthesis, Reto presented a unified framework for the generation of advanced light, including optical lattices. This work allows the creation of simpler microscope systems that can be more readily disseminated to the broader community. Reto has also addressed the challenges of cleared tissue imaging by creating a highresolution, refractive indexindependent light-sheet

Reto Fiolka, Assistant Professor, Department of Cell Biology, University of Texas Southwestern.

microscope that can image centimeter-sized tissue sections with isotropic, $300 \mathrm{~nm}$ resolution. Reto and his collaborators have also developed a quantitative method to analyze cellular shapes and to extract information about cellular morphology connected to signaling in health and disease. More recently, Reto's laboratory discovered a novel form of projection imaging, which is leveraged to view microscopic samples from different angles in real time. This invention improves sample exploration, as 3D specimens can be viewed from different angles at a video rate.

Reto's research has extended the imaging capabilities of optical microscopy such that cancer cell research and drug screening can be performed in physiologically relevant, $3 \mathrm{D}$ ex vivo and in vivo environments. The imaging tools developed by Reto are being used in collaborative research at UT Southwestern and across the United States.

\section{Burton Medal - Physical Sciences: Huolin Xin}

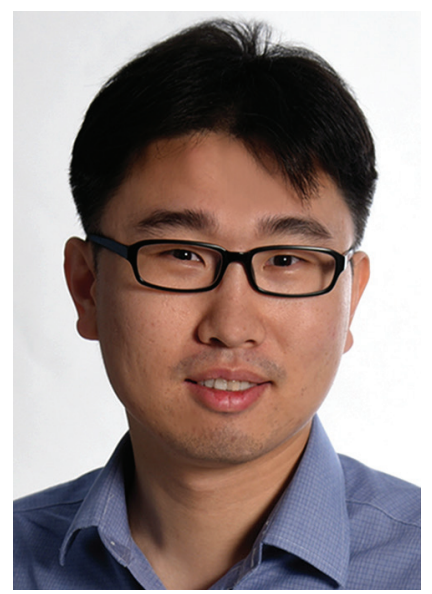

Huolin Xin graduated from the Physics Department of Cornell University in 2011 and joined the University of California, Irvine in 2018. As an early-career investigator, Huolin is a leading expert in the field of transmission electron microscopy, and he has many world-class contributions to the development of in situ three-dimensional analytical imaging, quantitative spectroscopy at the atomic scale, and artificially intelligent TEM. Using these tools, Huolin has resolved many important materials, physics, and chemistry prob-

Huolin Xin, Associate Professor, Department of Physics and Astron omy, University of California, Irvine.

lems resulting in energy technology advancements. In particular, Huolin is exceptionally productive and has a stunning 
publication record. In his short 7-year career, he has authored and co-authored over 240 peer-reviewed journal articles, 35 of which were published in Science and Nature sister journals. On 10 of the 35 , he is the senior author who led the research. His recent research finding on battery materials was selected as a 2014 Top 10 Scientific Achievement by Brookhaven National Lab. Even though Huolin has only worked at UC Irvine for two years, he has already built a robust research program in the Department of Physics and Astronomy. He has won three federal awards (DOE and NSF) and one industrial (Toyota) research grant as the PI, with a total worth of $\$ 3.63$ million.

Apart from his scientific and teaching achievements, Huolin has demonstrated great leadership in the microscopy community. He has organized multiple symposia and premeeting congresses, served as leader of the MSA Liquid and Gas TEM Focused Interest Group, and was Program Chair for the M\&M 2020 meeting.

\section{Albert Crewe Award}

The Albert Crewe Award was initiated to recognize annually the distinguished contributions to the field of microscopy and microanalysis in the physical sciences of an early career scientist, of not more than 6 years' standing since doctoral graduation, for research performed during this period.

\section{Albert Crewe Award: Wenpei Gao}

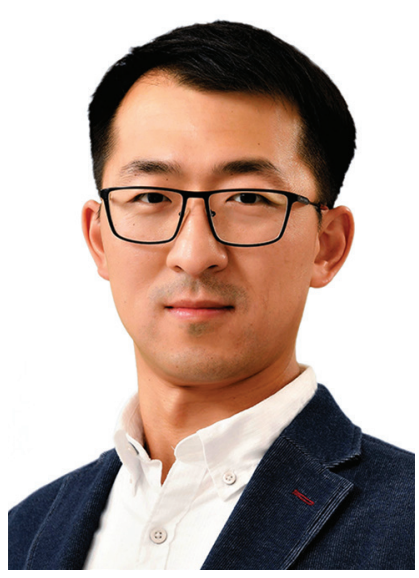

Wenpei Gao, Assistant Professor, Department of Materials Science and Engineering, North Carolina State University. nanostructured catalysts during nucleation, growth, and corrosion. His research has been recognized by multiple awards, including the Hammer Fellowship and Racheff-Inter Fellowship from the University of Illinois, and the Eric Samuel Scholarship and Postdoctoral Award from MSA. As a leading young microscopist and materials scientist, Wenpei has focused not only on advancing electron microscopy techniques, but also on applying these new approaches in insightful ways to tackle fundamental and critical questions in materials science.

Wenpei's research at NC State involves developing analytical and 4D STEM imaging techniques that can be applied to study a wide range of problems in materials, including the phase and polarization states at different scales in perovskite oxides and the structural properties of metalorganic-frameworks (MOF). He has also initiated the study of artificial intelligence (AI)-based image processing for in situ electron microscopy. Wenpei's research is taking full advantage of state-of-the-art electron microscopy and advancing the field of characterization sciences, bringing many new possibilities for interdisciplinary research.

\section{George Palade Award}

The George Palade Award was initiated to recognize annually the distinguished contributions to the field of microscopy and microanalysis in the life sciences of an early career scientist, of not more than 6 years' standing since doctoral graduation, for research performed during this period.

\section{George Palade Award: Yong Zi Tan}

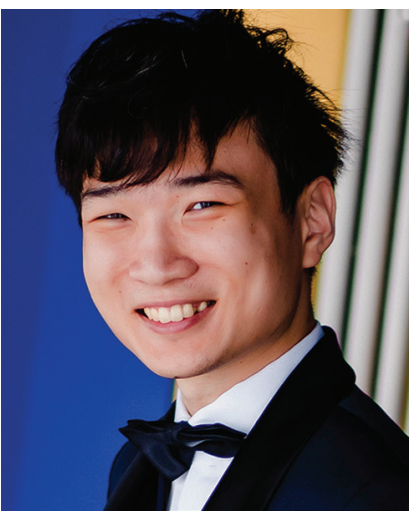

Yong Zi Tan, Assistant Professor, The National University of Singapore.
Yong $\mathrm{Zi}$ Tan obtained his BSc in Biochemistry from Imperial College London (UK) and earned his $\mathrm{PhD}$ from Columbia University, where he worked to solve challenging structures using single-particle cryo-EM and to develop cryo-EM methodologies. Occasionally a young scientist will become prominent even before they have started their own research group. Yong $\mathrm{Zi}$ is one of these rare early stars. His firstauthored Nature papers on tilting specimens to deal with preferred orientations and Ewald sphere correction to achieve extremely high resolution caught the attention of the field. These methods tackle difficult specimens for structural studies and point to how far cryo-EM will be able to go for high-resolution structure determination. Most cryo-EM experts specialize in either method development or biological application of the tool. However, Yong $\mathrm{Zi}$ produces world-class results in both areas. His results on mycobacterial and malarial protein structures cemented his reputation as a productive and accomplished scientist. These contributions represent highly soughtafter targets that may open the way for developing therapeutics against malaria and TB. Yong $\mathrm{Zi}$ has also made significant advances studying the mammalian V-type ATPase, a central enzyme in eukaryotic bioenergetics, by determining the conformations the protein complex adopts in different nucleotidebound states throughout its catalytic cycle. Yong Zi recently joined the National University of Singapore, where he will continue synergizing biological questions with technical methods development related to membrane proteins involved in human diseases.

\section{Chuck Fiori Award for Outstanding \\ Technologist in the Physical Sciences}

This award annually honors a technologist from the physical sciences who has made significant contributions, such as the 
development of new techniques that have contributed to the advancement of microscopy and microanalysis. A technologist is defined as an individual whose primary role is in microscopy and microanalysis tool development or service. Established principal investigators/project managers are not eligible for this award.

\section{Fiori Award: Karen Bustillo}

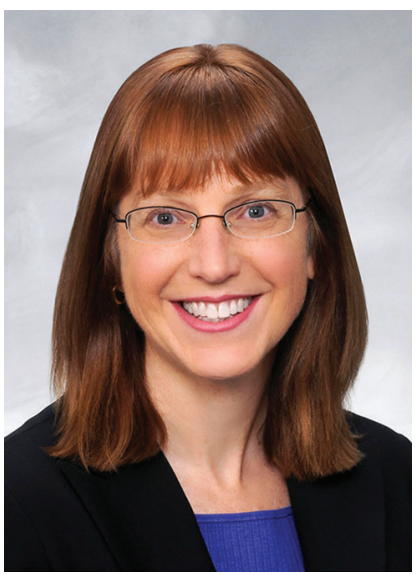

Karen Bustillo, Principal Scientific Engineering Associate, National Center for Electron Microscopy, Lawrence Berkeley Lab.

Karen Bustillo joined the Lawrence Berkeley National Center for Electron Microscopy ing her $\mathrm{PhD}$ in Materials Science and Engineering from UC Berkeley, where she used photoluminescence, Raman spectroscopy, and TEM to study electronic materials. She also has a strong background in solid-state NMR. Through the Molecular Founder user program at NCEM, Karen has worked with and contributed to the research of microscopists from around the world. At the NCEM, she manages (NCEM) in 2013 after completthree transmission electron microscopes with emphasis on STEM EDS, 4D-STEM, and in situ capabilities. She is also a highly sought-after mentor for new users and staff at LBL and has made a lasting impact in the EM community far beyond LBL. Last year, she was the scientific contact person for over 30 active projects from national and international groups (plus another $\sim 70$ users that use the three instruments she oversees on day-to-day operations). She is the scientific advisor on a portfolio of user projects that includes soft organic materials, inorganic nanoparticles, cosmic samples, and biological samples. With the NCEM team, she has pushed the development of scanning nano diffraction, or 4D-STEM, especially as it is applied to polymers, framework materials, and other beam-sensitive materials.

\section{Hildegard H. Crowley Award for Outstanding Technologist in the Biological Sciences}

This award annually honors a technologist from the biological sciences who has made significant contributions, such as the development of new techniques that have contributed to the advancement of microscopy and microanalysis. A technologist is defined as an individual whose primary role is in microscopy and microanalysis tool development or service. Established principal investigators/project managers are not eligible for this award.

\section{Crowley Award: Trace A. Christensen}

Trace is a Research and Development Specialist at the Mayo Clinic Microscopy and Cell Analysis Core Facility in Rochester, MN. He received a BS degree in Biology from Saint John's University and MS degree in Biotechnology Enterprise from Johns Hopkins University. Trace has also spent time training at MBL, University of Colorado Boulder, and the Scripps Research Institute. He has worked in biological microscopy and research

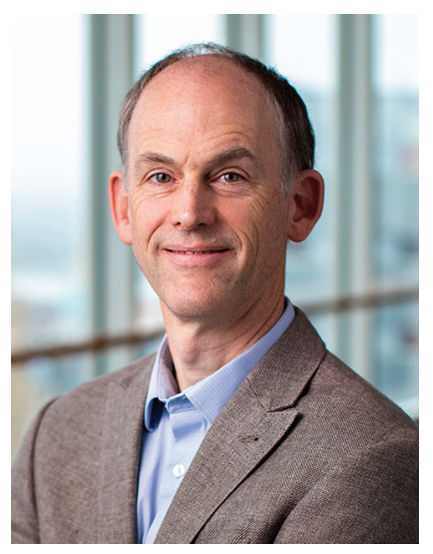

for over thirty years at the Mayo Clinic. He is involved in designing experiments and developing protocols to facilitate the research goals of investigators using a variety of imaging modalities. He has extensive experience in microwave processing and 3DEM techniques. Recently, he contributed significantly to work in mitochondrial dynamics of Alzheimer's disease, diabetes, Usher syndrome, and ischemic strokes. Trace enjoys mentor-

Trace A. Christensen, Mayo Clinic Microscopy and Cell Analysis Core. ing both undergrad and graduate students. He helped design and build a 3DEM service at the Mayo Clinic that includes processing, serial block-face imaging, and data analysis that is utilized by researchers at the Mayo Clinic and throughout the country. Trace has been an active member of MSA since 2003 and served as Treasurer of the Diagnostic and Biomedical Microscopy Focused Interest Group for several years. He is also a member of the Minnesota Microscopy Society and is actively working to help bring new microscopy and data analysis technologies to local universities and research institutions.

\section{Morton D. Maser Distinguished Service Award}

This award recognizes outstanding volunteer service to the Microscopy Society of America as exemplified by Mort Maser, who served the Society for many years with great dedication. This award is made annually to honor an MSA member who has provided significant volunteer service to the Society over a sustained period of time.

\section{Morton D. Maser Award: Leona Cohen-Gould}

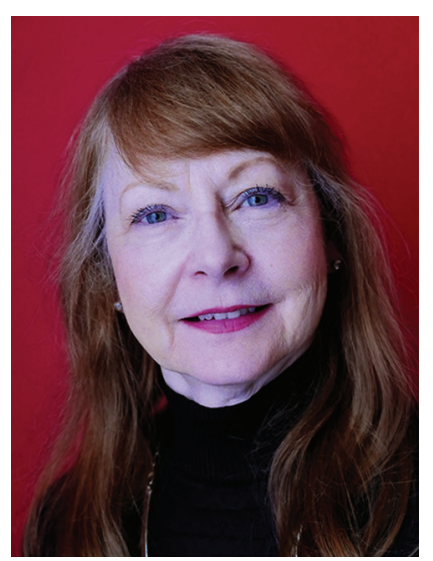

Lee has been an active member of (E)MSA since 1980 and has attended the annual $M \& M$ meeting since 1998. She is a member of the Certification Board and the Technologists' Forum, and she was Chair of the Certification Board from 2011-2013, Vice-Chair from 2015-2018, and is once again serving as Chair, 2019-2021. In her role as Chair, Lee coordinates the biannual exam cycle to certify Biological EM Technologists.

Leona Cohen-Gould, Senior Staff Associate in Biochemistry, Microscopy, and Image Analysis, and Core Co-Director, Electron Microscopy and Optical Microscopy Cores, Weill Cornell Medical College, New York. She reviews the applicants for eligibility, arranges for a proctor, and administers the written exam. She collects the submissions for the practical exam and distributes the material to other Board members for evaluation and grading. She then notifies the candidate of the results. Lee regularly 
interacts with the Business Office to carry out these duties. Lee has actively reviewed the content of the written exam and, with the assistance of the other Board members, regularly updates the exam questions. Lee joined the Technologists' Forum in 2010, served as its Vice-Chair from 2014-2017, and is currently a Board member. In these roles she has assisted in symposium topic selection and recommendations for speakers. Lee has also participated as an invited speaker for several Technologists' Forum symposia. Lee is also involved in her Local Affiliated Society, the New York Society of Experimental Microscopists (NYSEM), where she has also served in leadership roles as Treasurer and Secretary.

\section{MSA Fellows}

The designation MSA Fellow is intended to recognize those who have been conferred the Society's Distinguished Scientist Awards, as well as senior distinguished members of the Society who have been a member of the Society for at least 10 years and have made significant contributions to the advancement of the field of microscopy and microanalysis through a combination of scientific achievement, service to the scientific community, and the Society itself. Election to MSA Fellow is highly selective and represents a broad cross-section of the MSA membership. The number of MSA members elected to Fellowship each year is restricted to no more than $0.5 \%$ of the total MSA membership. For each Fellow, the nominating statement supporting their award is italicized, followed by a short summary of their service to the MSA or microscopy research community.

\section{MSA Fellows with Nominating Statement}

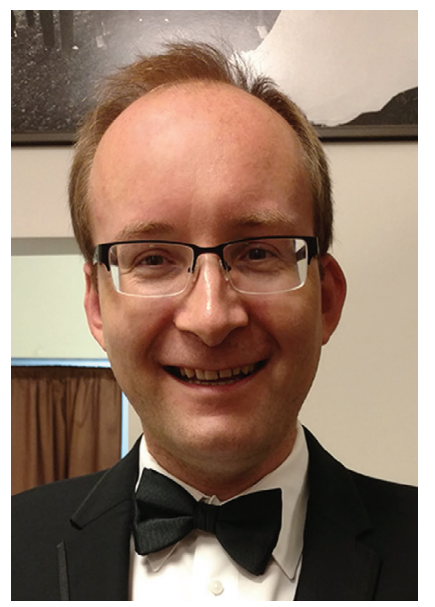

Sergei Kalinin, Corporate Fellow, Center for Nanophase Materials Sciences, Oak Ridge National Laboratory.

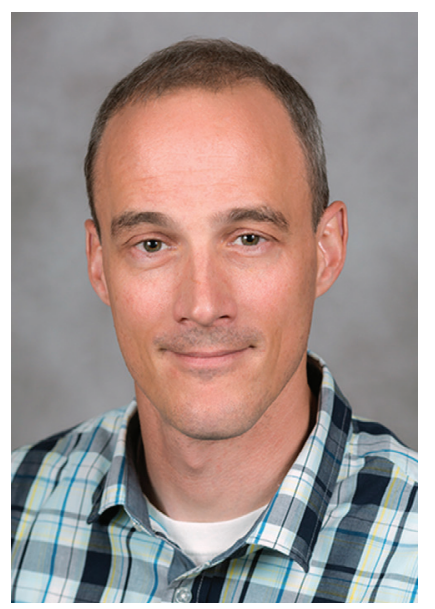

Robert Klie, Assistant Professor of Physics, University of Illinois Chicago.
Sergei Kalinin: For pioneering contributions to quantitative scanning probe and scanning transmission electron microscopy, including functional imaging, machine learning, and atomic fabrication. In 2015, Sergei received the Royal Microscopical Society Medal for Scanning Probe Microscopy, Senior membership of IEEE, and Fellowships of both the American Vacuum Society and the American Physical Society. $\mathrm{He}$ achieved Fellowship in the Materials Research Society in 2017. He has 670 publications with an h-index of 90 with over 24,000 citations and an average of 36 citations per publication.

Robert Klie: For his essential contributions to the fields of atomic-resolution scanning transmission electron microscopy, electron energyloss spectroscopy, and in situ heating/cooling techniques solving materials problems in oxide thin-films, catalysts, and superconductors. Robert has received several awards as a graduate student (MRS Gold Award, MSA Presidential Award), Postdoctoral Research Associate (Goldhaber Distinguished Fellowship), and faculty member (NSF Career Award, UIC
Excellence in Teaching and Learning). The more than 12,000 citations of his research are indicative of the high quality of his work.

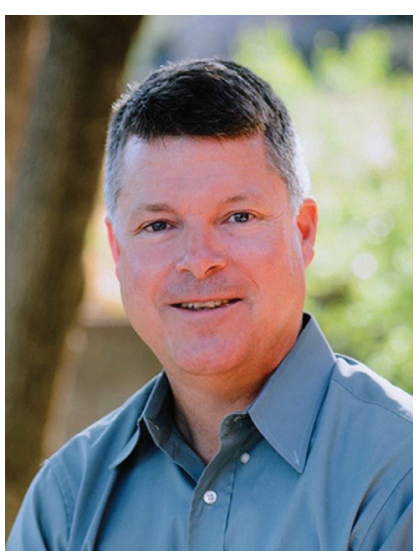

Paul Kotula, Principal Member of Technical Staff, Sandia National Laboratories.

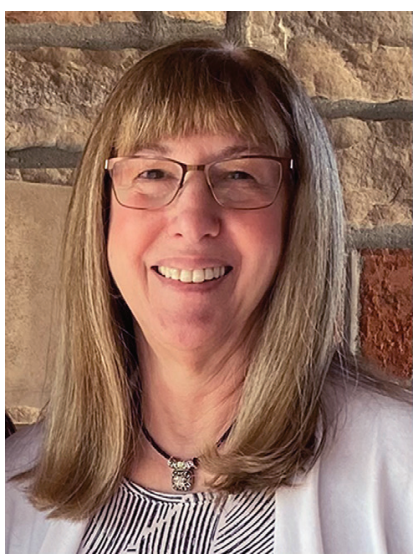

Pamela Lloyd, Research Scientist (Retired), UES, Inc.
Paul Kotula: For contributions to the field of statistical analysis of spectral data and dedicated long-term service to the Microscopy Society of America. Paul's work has achieved an h-index of 33 with more than 300 citations per year. In addition to his excellent research reputation, Paul has been a long-time volunteer for MSA, having served as Program Chair in 2006, MSA Director from 2014-2016, and President in 2019.

Pamela Lloyd: For being a distinguished, long-standing member of the Society, serving key roles on numerous committees and in elected positions, as well as for her expert contributions in biofilms and microbial contamination. Pam has been an MSA member for 40 years and has served in many capacities within the Society, including Secretary on the Executive Council for 2 terms, Physical Sciences Director (2009-2011), MegaBooth Coordinator, Placement 
Office Chair, Education Committee member, Technologists' Forum member, Traveling Poster Coordinator, and Organizing Committee for M\&M 1993 in Cincinnati. Pam received the Morton D. Maser Distinguished Service Award in 2010.

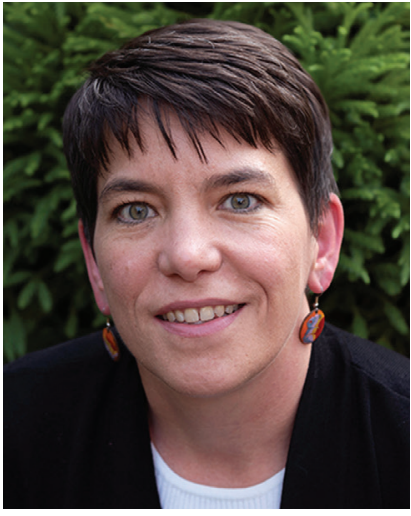

Rhonda Stroud, Head, Nanoscale Materials Section, US Naval Research Laboratory.
Rhonda Stroud: For her outstanding contributions and sustained leadership in advancing our knowledge and understanding of extraterrestrial materials, especially using aberration-corrected electron microscopy and vibrational spectroscopy. Rhonda's studies of extra-terrestrial materials using vibrational spectroscopy are particularly noteworthy, and she has demonstrated the possibility of detecting single (isotopic) atoms, which are important

indicators of primordial origins. She has published more than 180 research papers with an h-index of 57 with nearly 12,500 citations.

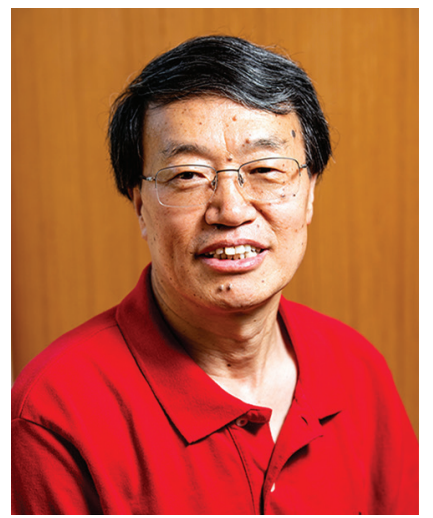

Chongmin Wang, Lab Fellow and Materials Scientist, Pacific Northwest National Laboratory.
Chongmin Wang: For being a pioneer in developing in situ TEM tools for studying energy storage materials under dynamic operating conditions, leading to new understanding of charge transport and structural changes in materials. Chongmin was the recipient of the 2012 Microscopy Today Innovation Award for developing an in situ TEM technique for battery research and has over 390 publications, an h-index of 83 , and approximately 19,000 citations.

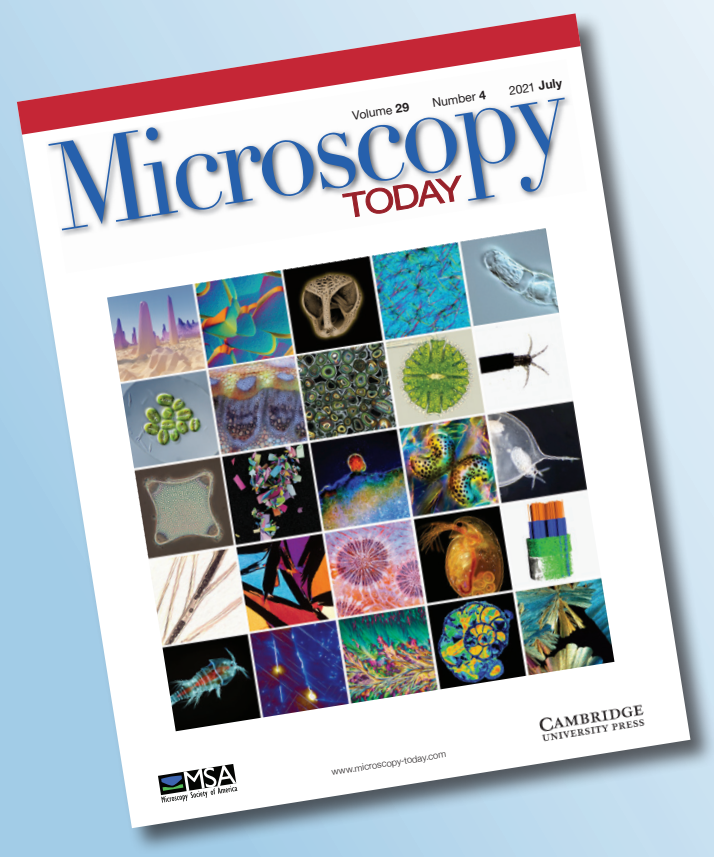

July cover showing the 25 finalists of the 2021 competition

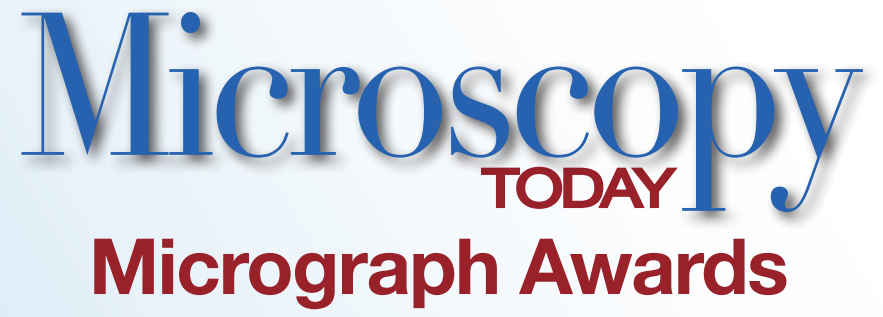

To submit your micrographs for the 2022 competition go to the following website:

$$
\begin{aligned}
& \text { https://www.microscopy.org/awards/ } \\
& \text { micrograph_competition.cfm }
\end{aligned}
$$

Deadline for the next competition: February 22, 2022 